Artigo Original

\title{
Ocorrência de neutropenia em mulheres com câncer de mama durante tratamento quimioterápico*
}

\author{
Occurrence of neutropenia in women with breast cancer during chemotherapy treatment
}

Ocurrencia de neutropenia en mujeres con cáncer de mama durante el tratamiento quimioterápico

\section{RESUMO}

Objetivo: Analisar a ocorrência de neutropenia induzida por drogas utilizadas no tratamento quimioterápico de mulheres com câncer de mama. Métodos: Estudo retrospectivo, com avaliação de 72 prontuários, durante 2003-2006. Resultados: Dos 558 ciclos de quimioterapia realizados, foram registrados 152 eventos adversos nos períodos de neoadjuvância e adjuvância, totalizando 43 casos por toxicidade hematológica. Quanto à ocorrência de neutropenia, $43 \%$ apresentaram, pelo menos, um episódio durante o tratamento. Testes de hipótese para comparar as médias dos valores de glóbulos brancos entre as mulheres que apresentaram ou não neutropenia apontaram para valores estatisticamente significantes, nos ciclos dois e três da neoadjuvância e nos ciclos dois, três e quatro da adjuvância. Conclusão: A neutropenia, tanto na neoadjuvância como na adjuvância ocorreu a partir do segundo ciclo e manteve-se durante o tratamento e foi estatisticamente significante quando foram compadas as mulheres que tiveram ou não esta ocorrência.

Descritores: Quimioterapia/efeitos adversos; Neutropenia; Neoplasias da mama/quimioterapia

\begin{abstract}
Objective: To analyze the occurrence of neutropenia induced by drugs used in chemotherapy treatment for women with breast cancer. Methods: Retrospective study, with assessment of 72 records, between 2003-2006. Results: Of the 558 cycles of chemotherapy performed, there were 152 adverse events registered in adjuvant and neoadjuvant therapy periods, totaling 43 cases of haematological toxicity. Regarding the occurrence of neutropenia, $43 \%$ had at least one episode during treatment. Hypothesis tests to compare the mean values of white blood cells from women who did or did not present with neutropenia pointed to statistically significant values in cycles two and three of neoadjuvant therapy and in cycles two, three and four in the adjuvant therapy. Conclusion: Neutropenia in both the adjuvant and neoadjuvant therapy occurred beginning with the second cycle and was maintained during treatment, and was statistically significant when compared women who had to those who did not have this occurrence.
\end{abstract}

Keywords: Drug therapy/adverse effects, Neutropenia; Breast neoplasm/drug therapy

\section{RESUMEN}

Objetivo: Analizar la ocurrencia de neutropenia inducida por drogas utilizadas en el tratamiento quimioterápico de mujeres con cáncer de mama. Métodos: Estudio retrospectivo, realizado con la evaluación de 72 historias clínicas, durante loa años 2003-2006. Resultados: De los 558 ciclos de quimioterapia realizados, fueron registrados 152 eventos adversos en los períodos de neoadyuvante y adyuvante, totalizando 43 casos por toxicidad hematológica. En cuanto a la ocurrencia de neutropenia, el $43 \%$ presentaron, por lo menos, un episodio durante el tratamiento. Las pruebas de hipótesis para comparar los promedios de los valores de glóbulos blancos entre las mujeres que presentaron o no neutropenia apuntaron hacia valores estadísticamente significativos, en los ciclos dos y tres de la neoadyuvancia y en los ciclos dos, tres y cuatro de la adyuvancia. Conclusión: La neutropenia, tanto en la neoadyuvancia como en la adyuvancia ocurrió a partir del segundo ciclo y se mantuvo durante el tratamiento y fue estadísticamente significativa cuando fueron compadas las mujeres que tuvieron o no esta ocurrencia. Descriptores: Quimioterapia/efectos adversos, Neutropenia, Neoplasias de la mama/quimioterapia

* Trabalbo realizado no Hospital das Clinicas da Faculdade de Medicina de Ribeirão Preto, Universidade de São Paulo - USP - Ribeirão Preto (SP), Brasil. ${ }^{1}$ Doutora em Enfermagem. Professora do Departamento Materno-Infantil e Saúde Pública da Escola de Enfermagem de Ribeirão Preto, Universidade de São Paulo - USP - Ribeirão Preto (SP), Brasil.

${ }^{2}$ Enfermeira. Pós-graduanda (Mestrado) do Programa de Pós-graduação Enfermagem em Saúde Pública da Escola de Enfermagem de Ribeirão Preto, Universidade de São Paulo - USP - Ribeirão Preto (SP), Brasil.

3 Doutora em Enfermagem. Professora do Departamento Materno-Infantil e Saúde Pública da Escola de Enfermagem de Ribeirão Preto, Universidade de São Paulo - USP - Ribeirão Preto (SP), Brasil.

${ }^{4}$ Livre-docente em Enfermagem. Professora Associada do Departamento Materno-Infantil e Saúde Pública da Escola de Enfermagem de Ribeirão Preto, Universidade de São Paulo - USP - Ribeirão Preto (SP), Brasil.

Autor Correspondente: Thaís de Oliveira Gozzo

Artigo recebido em 28/10/2010 e aprovado em 07/06/2011

Av. Bandeirantes, 3900 - Monte Alegre - Ribeirão Preto - SP - Brasil

Cep: 14040-902 E-mail: thaisog@eerp.usp.br 


\section{INTRDUÇÃO}

Um aumento na sobrevida de mulheres com neoplasia mamária tem sido observado, e as taxas de mortalidade vêm diminuindo cerca de $2,3 \%$ por ano, em razão da ampla participação nos programas de rastreamento com maior proporção de pacientes diagnosticadas em estágio inicial da doença e em decorrência do sucesso da crescente utilização de regimes adjuvantes de poliquimioterapia e anticorpos monoclonais ${ }^{(1)}$.

Embora estes agentes sejam projetados para localizar e eliminar as células neoplásicas, seus efeitos citotóxicos, frequentemente, afetam as células normais, resultando em eventos adversos indesejados. Mesmo os medicamentos mais recentes e as novas variações de combinações apresentam propriedades adversas, seja em maior ou menor grau, o que reforça a necessidade do uso racional de drogas para evitar os efeitos adversos acumulativos ${ }^{(2-3)}$.

A mielossupressão, em especial, a neutropenia, é o evento adverso mais temido durante a quimioterapia antineoplásica, pois aumenta a morbidade e a mortalidade entre os indivíduos nesse regime terapêutico. Tal evento interfere diretamente no limite da dosagem do quimioterápico, aumenta os custos do tratamento, além de não ser possível prever quais pacientes irão apresentá-la ${ }^{(4-5)}$.

A neutropenia induzida por quimioterapia pode levar à neutropenia febril, que requer hospitalização imediata e tratamento com antibióticos em razão do risco aumentado de infecções e, consequentemente, maior risco de vida. A taxa de mortalidade hospitalar associada a pacientes que são internados por neutropenia febril é de 9,5\%, em média, e mais de $21 \%$ para aqueles que possuem comorbidades ${ }^{(6)}$.

A neutropenia pode ser definida baseada nos resultados dos testes laboratoriais que indicam uma diminuição no número de glóbulos brancos (GB) em uma amostra de sangue. Pode ser classificada em cinco graus, a saber: grau I valores de $\mathrm{GB}=3 \times 10^{9}$ células $/ \mathrm{L}$, grau II, de $2-3 \times 10^{9}$ células/L; grau III, de $1-2 \times 10^{9}$ células/L; grau IV, $<1.000 / \mu \mathrm{L}$ e grau $\mathrm{V}$, indicativo de óbito para o indivíduo no regime terapêutico ${ }^{(7)}$

Estudos a respeito da quimioterapia neoadjuvante, combinando docetaxel e epirrubicina, destacam que a neutropenia ocorre com uma frequência de $6 \%$ a $81 \%$ e a neutropenia febril de $1 \%$ a $22 \%{ }^{(8-10)}$. Quanto aos eventos adversos dos esquemas de quimioterapia adjuvante combinando ciclofosfamida e epirrubicina, em estudo para tratamento de câncer de mama metastático, observou-se uma incidência de neutropenia de graus III/IV em 45\% das participantes e $2 \%$ de neutropenia febril ${ }^{(11)}$.

Considera-se que o conhecimento dos eventos adversos e das alternativas de controle e prevenção são indispensáveis para o manejo de pacientes oncológicos em tratamento quimioterápico. Assim, a identificação da neutropenia, como evento de importância vital deve ser foco do cuidado de enfermagem, e a partir desta identificação a adoção de medidas de proteção e estratégias de educação em saúde dirigida à mulher e familiares deve fazer parte do plano de cuidados. O cuidado inclui orientações sobre tais eventos relacionados ao tratamento quimioterápico e deve estar fundamentado em informações assimiláveis, reforçando assim os benefícios das drogas e das alternativas voltadas ao manejo dos possíveis eventos adversos.

Diante do exposto, o objetivo deste estudo consistiu em analisar a ocorrência de neutropenia induzida por drogas utilizadas no tratamento quimioterápico de mulheres com câncer de mama

\section{MÉTODOS}

Um estudo de corte transversal foi realizado com o uso de dados secundários coletados dos prontuários de mulheres com câncer de mama, atendidas no Ambulatório de Mastologia do Hospital das Clínicas da Faculdade de Medicina de Ribeirão Preto da Universidade de São Paulo, durante os anos de 2003 e 2006. O estudo foi aprovado pelo Comitê de Ética em Pesquisa da instituição sob o número 8.602/2007 que dispensou a solicitação do Termo de Consentimento Livre e Esclarecido.

O protocolo de quimioterapia utilizado no período estudado consistia, preferencialmente, no uso de Epirrubicina $50 \mathrm{mg} / \mathrm{m}^{2}$ e Docetaxel $75 \mathrm{mg} / \mathrm{m}^{2}$ para o tratamento neoadjuvante e, para o adjuvante, Ciclofosfamida $600 \mathrm{mg} / \mathrm{m}^{2}$ e Epirrubicina $60 \mathrm{mg} / \mathrm{m}^{2}$. Por essa razão, os esquemas foram selecionados.

Após a leitura de 404 prontuários, foram selecionados 72 que pertenciam a mulheres que preenchiam os critérios de inclusão: faixa etária de 30 a 60 anos; tratamento quimioterápico como primeira opção do tratamento, seguido de complementação na adjuvância; uso dos esquemas quimioterápicos de neoadjuvância e adjuvância propostos para este estudo; tratamento quimioterápico finalizado até dezembro de 2006.

Para a coleta de dados, elaborou-se um instrumento com informações sobre: identificação, diagnóstico, resultados dos hemogramas realizados antes de cada ciclo de quimioterapia, dosagem dos quimioterápicos utilizados na neoadjuvância e na adjuvância, internações durante o tratamento quimioterápico e sobre o procedimento cirúrgico.

Realizou-se análise descritiva das variáveis sóciodemográficas e contagem de GB. Para a variável GB, foi feita a análise descritiva para cada ciclo em dois períodos: neoadjuvante (cinco ciclos) e adjuvante (seis ciclos). A ocorrência de neutropenia foi definida com base no valor de GB avaliado por meio do hemograma realizado antes da administração da quimioterapia, considerando os valores de $\mathrm{GB} \leq 3 \times 10^{9}$ para a definição da neutropenia ${ }^{7}$.

\section{RESULTADOS}

A análise dos prontuários mostrou que 28\% das mulheres tinham idade entre 51 e 55 anos; predomínio da cor branca $(83,3 \%)$ e da mama esquerda comprometida (58\%). O estádio clínico do câncer de mama mais observado foi o IIIa, com uma frequência de 34,7\% das mulheres, seguido do IIIb em 27,7\% (Tabela 1).

Durante o tratamento quimioterápico, foram realizados 558 ciclos, com registro de intercorrências 
variadas, que interferiram na duração do tratamento das mulheres estudadas. Foram registrados 152 eventos adversos nos prontuários avaliados nos períodos de neoadjuvância e adjuvância, destes, 18 e 25 foram por toxicidade hematológica, respectivamente. Ressalta-se que uma mesma mulher pode apresentar mais que um evento no mesmo ciclo e durante todo o tratamento. Algumas mulheres foram internadas para tratamento de eventos adversos, e que a maioria foi por neutropenia febril (Tabela 2)

Tabela1 - Mulheres submetidas à quimioterapia para câncer de mama no período de 2003 a 2006, conforme cor, lateralidade do tumor, estádio clínico, tipo de neoplasia e cirurgia realizada. Ribeirão Preto, 2008

\begin{tabular}{lc}
\hline Características & $\begin{array}{c}\text { Núme ro e porcentagem } \\
(\%) \text { de mulheres }\end{array}$ \\
\hline Cor & $60(83,3)$ \\
B ranca & $2(2,7)$ \\
Negra & $10(14)$ \\
P arda & \\
Lateralidade do tumor & $42(58)$ \\
Es querdo & $30(42)$ \\
Direito & $12(16,6)$ \\
Estádio da doença & $15(21)$ \\
II & $25(34,7)$ \\
IIb & $20(27,7)$ \\
IIIa & \\
IIIb & $51(71)$ \\
Tipo de neoplasia & $14(19,3)$ \\
Ductal invasor & $4(5,5)$ \\
Ductal in filtrante & $3(4,2)$ \\
Lobular & \\
Outros & $29(40,3)$ \\
Tipo de cirurgia & $41(57)$ \\
Ciru rgia radical & $2(2,7)$ \\
Ciru rgia conservadora & \\
Sem cirurgia & \\
\hline
\end{tabular}

Assim, em relação à ocorrência de neutropenia, observou-se que 31 mulheres (43\%) apresentaram, pelo menos, um episódio durante o tratamento, seja na neoadjuvância ou na adjuvância.

Os valores de GB na neoadjuvância variaram de $0,4 \times 10^{9}$ a $13,8 \times 10^{9}$ células/L naquelas mulheres que apresentaram neutropenia e de 3,5 a $16,4 \times 10^{9}$
Tabela 2 - Mulheres submetidas à quimioterapia para câncer de mama no período de 2003 a 2006, conforme intercorrências entre os ciclos. Ribeirão Preto, 2008 $(\mathrm{N}=558)$

\begin{tabular}{lccc}
\hline Intercorrências & Neoadjuvante & Adjuvante & Total \\
\hline Número de ciclos & 253 & 305 & 558 \\
Número total de & 77 & 75 & 152 \\
$\begin{array}{l}\text { eventos adversos } \\
\text { Toxicida de não }\end{array}$ & 59 & 50 & 109 \\
h ematológica & & & \\
Toxicida de & 18 & 25 & 43 \\
hematológica & & & \\
Número total de & 14 & 5 & 19 \\
hospitalizações & & & \\
$\begin{array}{l}\text { Neutropenia febril } \\
\text { Outros motivos }\end{array}$ & 13 & 2 & 15 \\
\hline
\end{tabular}

células/L entre as que não apresentaram. O Teste de hipótese para a comparação entre as médias dos grupos com e sem neutropenia aponta para valores estatisticamente significativos para GB antes de iniciar o tratamento nos ciclos dois e três. Os ciclos quatro e cinco não foram considerados, pois o número de mulheres submetidas a estes ciclos foi menor que nos outros (Tabela 3).

$\mathrm{Na}$ adjuvância, quando considerados os quatro primeiros ciclos, em que se concentra a maioria das participantes, o p-valor das médias dos resultados dos GB para os grupos com e sem neutropenia mostrou diferença estatisticamente significante para os ciclos dois, três e quatro, como exposto nos dados da Tabela 4 .

No presente estudo, a média de idade entre as mulheres que não apresentaram neutropenia foi de 48,5 anos, e àquelas que apresentaram, foi de 46,7 anos e não se encontrou diferença estatisticamente significativa na análise desta variável $(p=0,2955)$.

Durante o tratamento quimioterápico, das 72 mulheres estudadas, 16 foram internadas, uma ou mais vezes, em decorrência de eventos adversos da quimioterapia ou por complicações cirúrgicas, totalizando 19 internações. Em relação ao período de tratamento, 14 internações ocorreram durante o tratamento neoadjuvante, quatro por complicações da cirurgia, antes de iniciar o tratamento adjuvante, e cinco no tratamento adjuvante.

Os motivos das internações foram variados, com

Tabela 3 - Estatísticas descritivas da variável GB entre as mulheres que apresentaram ou não neutropenia, durante a quimioterapia neoadjuvante, no período de 2003 a 2006. Ribeirão Preto, 2008 ( $n=72)$

\begin{tabular}{|c|c|c|c|c|c|c|c|c|c|}
\hline Neutropenia neoad juvante & $\mathrm{n}^{\circ}$ & V ari ável & $\mathbf{n}^{\circ}$ & Média & Median a & D es vio-Pad rão & Mínimo & Máximo & Valorde p \\
\hline \multirow[t]{5}{*}{ N ão } & 41 & GBN1 & 41 & 8,0 & 7,8 & 2.2 & 4,5 & 16,4 & 0,0166 \\
\hline & & GBN2 & 41 & 8,5 & 8,2 & 2,7 & 3,7 & 14,4 & 0,0016 \\
\hline & & GBN3 & 39 & 8,5 & 8,0 & 2,7 & 3,8 & 14,4 & 0,0009 \\
\hline & & GBN4 & 19 & 7,5 & 6,5 & 2,8 & 3,5 & 14,4 & 0,1663 \\
\hline & & GBN5 & $3^{*}$ & 6,3 & 6,5 & 1,2 & 5,1 & 7,5 & - \\
\hline \multirow[t]{5}{*}{$\operatorname{Sim}$} & 31 & GBN1 & 31 & 6,8 & 6,7 & 1,9 & 3,4 & 11,2 & \\
\hline & & GBN2 & 31 & 6,2 & 7,0 & 3,1 & 0,4 & 11,3 & \\
\hline & & GBN3 & 29 & 6,2 & 5,8 & 2,8 & 1,3 & 12,3 & \\
\hline & & GBN4 & 15 & 6,2 & 6,0 & 2,8 & 1,0 & 13,8 & \\
\hline & & GBN5 & 5 & 3,5 & 1,9 & 2,3 & 1,8 & 6,7 & \\
\hline
\end{tabular}

* não foi possível realizar o teste comparativo

p-valor obtido da comparação: H0: a média das pacientes com neutropenia é igual a média das pacientes sem neutropenia, H1: são diferentes. Utilizou-se teste paramétrico adequado de acordo com o teste de igualdade de variâncias. 
Tabela 4 - Estatísticas descritivas da variável GB entre as mulheres que apresentaram ou não neutropenia durante a quimioterapia adjuvante, no período de 2003 a 2006. Ribeirão Preto, 2008 (n=72)

\begin{tabular}{|c|c|c|c|c|c|c|c|c|c|}
\hline Neutropenia adjuvante & $\mathbf{n}$ & Variável & n & Média & Mediana & Desvio-Padrão & Mínimo & Máximo & Valor de $p$ \\
\hline \multirow[t]{8}{*}{ Não } & 41 & GBPRÉ* & 40 & 7,8 & 7,7 & 24 & 3,5 & 15,0 & $<0,001$ \\
\hline & & GBA1 & 40 & 7,7 & 7,4 & 1,9 & 4,1 & 11,4 & 0,8075 \\
\hline & & GBA2 & 40 & 5,8 & 5,2 & 27 & 3,1 & 17,1 & 0,0014 \\
\hline & & GBA3 & 40 & 6,0 & 5,6 & 20 & 3,0 & 11,8 & 0,0030 \\
\hline & & GBA4 & 40 & 5,6 & 5,0 & 1,8 & 3,3 & 10,6 & 0,0163 \\
\hline & & GBA5 & $8^{* *}$ & 5,1 & 5,0 & 1,2 & 3,2 & 7,5 & - \\
\hline & & GBA6 & $7^{* *}$ & 5,0 & 4,5 & 20 & 3,0 & 9,5 & - \\
\hline & & GBPÓS*** & 39 & 6,20 & 5,69 & 2,90 & 3,20 & 20,79 & 0,0004 \\
\hline \multirow[t]{8}{*}{ Sim } & 31 & GBPRÉ & 30 & 5,4 & 6,3 & 23 & 0,8 & 9,3 & \\
\hline & & GBA1 & 30 & 7,5 & 6,5 & 3,4 & 3,6 & 20,8 & \\
\hline & & GBA2 & 30 & 4,0 & 3,5 & 1,8 & 1,1 & 7,9 & \\
\hline & & GBA3 & 30 & 4,4 & 3,7 & 1,9 & 2,3 & 10,6 & \\
\hline & & GBA4 & 30 & 4,4 & 3,9 & 21 & 1,7 & 12,3 & \\
\hline & & GBA5 & $5^{*}$ & 3,6 & 3,5 & 0,9 & 2,5 & 5,0 & \\
\hline & & GBCA6 & $3^{*}$ & 4,8 & 4,7 & 0,2 & 4,7 & 5,2 & \\
\hline & & GBPOS & 30 & 4,1 & 4,0 & 1,5 & 1,7 & 8,4 & \\
\hline
\end{tabular}

predomínio de neutropenia febril, que foi responsável por 15 das 19 internações, sendo 13 no tratamento neoadjuvante e duas no adjuvante.

Embora a neutropenia seja um motivo que, frequentemente, leva a atrasos nos ciclos da quimioterapia; neste estudo, observou-se que a maioria dos atrasos ocorreu em razão de outras ocorrências. Os motivos variaram e estão apresentados na Tabela 5 .

Tabela 5 - Mulheres submetidas à quimioterapia para câncer de mama no período de 2003 a 2006, conforme o motivo do atraso entre os ciclos, Ribeirão Preto, 2008 ( $n=153$ )

\begin{tabular}{|c|c|c|c|c|c|}
\hline \multirow{2}{*}{ Motivo do atraso } & \multicolumn{2}{|c|}{ Ne oadjuvant e } & \multicolumn{2}{|c|}{ Adjuv ante } & \multirow{2}{*}{$\begin{array}{c}\text { Total } \\
\text { Número }\end{array}$} \\
\hline & $\mathrm{n}^{\mathbf{o}}$ & $\%$ & $n^{\circ}$ & $\%$ & \\
\hline Não colheu exames & 5 & 13,1 & 6 & 5,2 & 11 \\
\hline Alteração nos exames & 14 & 38,5 & 67 & 58,2 & 82 \\
\hline Falta & 2 & 5,2 & 7 & 6,1 & 9 \\
\hline F al ta devaga & 3 & 8 & 3 & 2,6 & 6 \\
\hline Óbito & 2 & 5,2 & - & - & 2 \\
\hline Não relat ado & 8 & 21 & 20 & 17,4 & 28 \\
\hline Outros & 3 & 8 & 12 & 10,5 & 15 \\
\hline Total & 37 & 100 & 115 & 100 & 153 \\
\hline
\end{tabular}

\section{DISCUSSÃO}

A ocorrência de eventos adversos no tratamento quimioterápico é frequente e a neutropenia é um dos mais temidos. No tratamento do câncer de mama, observa-se que os protocolos em uso são considerados bem tolerados ${ }^{(8-10,12)}$, embora este estudo tenha encontrado cerca de $43 \%$ dessa ocorrência entre as mulheres estudadas.

A taxa apresenta-se condizente com estudos internacionais que têm buscado investigar tal evento adverso, destacando-se a escassez de estudos nacionais, o que dificulta comparações desta ocorrência com os protocolos implantados no Brasil.

Um estudo com 50 mulheres em uso de epirrubicina e docetaxel encontrou que $32 \%$ delas apresentaram neutropenia graus III/IV ${ }^{(9)}$. Outros estudos com as mesmas drogas apontam para a ocorrência de neutropenia grau IV, variando de 16\% a $81 \%{ }^{(10,12)}$. No protocolo neoadjuvante aqui estudado com as mesmas drogas também observouse a ocorrência de toxicidade em graus III e IV, e no ciclo dois, houve o valor mínimo de GB com diferença significativa estatisticamente entre as mulheres que apresentaram ou não a neutropenia.

Em estudo com uso de epirrubicina e ciclofosfamida (EC), encontrou-se uma incidência de neutropenia de graus III/IV em 16\% das participantes que utilizavam o regime padrão desses quimioterápicos (quatro ciclos de epirrubicina $90 \mathrm{mg} / \mathrm{m}^{2}$ e ciclofosfamida $600 \mathrm{mg} / \mathrm{m}^{2}$ no dia 1, a cada 21 dias), e dessas, foi observado em 5\% o atraso de uma semana no tratamento. No mesmo estudo randomizado de Fase II, foram avaliadas a segurança e a toxicidade relativa de regimes contendo doxorrubicina e ciclofosfamida (AC) versus epirrubicina e ciclofosfamida (EC), decorrente de regimes convencionais ou acelerados, em que a frequência da administração é aumentada sem alterar a dose total ou a duração. A ocorrência da neutropenia graus III/ IV foi observada em maior frequência no tratamento com os regimes padrões de ambos os protocolos quimioterápicos em relação aos acelerados ${ }^{(13)}$. No estudo aqui apresentado, observa-se a ocorrência de neutropenia graus II e III nos ciclos dois a quatro com diferença significativa estatisticamente.

Neste estudo, a ocorrência de neutropenia, aponta para a necessidade de a enfermagem definir protocolos de avaliação e seguimento, incluindo os efeitos colaterais e os graus de comprometimento, bem como seus manejos.

A implantação de protocolos propicia um controle mais eficiente na identificação dos eventos adversos e no manejo dos mesmos, favorecendo uma recuperação mais rápida das mulheres. Também evitam-se atrasos entre os ciclos e eventuais reduções de doses, que 
podem comprometer a resposta ao tratamento, além de propiciar a resistência tumoral aos quimioterápicos.

A neutropenia predispõe a ocorrência de neutropenia febril, que é uma complicação potencialmente fatal da quimioterapia, necessitando de hospitalização para tratamento empírico com antibióticos de amplo espectro, que pode levar ainda a redução da dose do quimioterápico ou atraso no tratamento, o que irá comprometer sua resposta ${ }^{(4-5)}$.

Um fator que pode interferir na ocorrência de neutropenia em pacientes durante o tratamento quimioterápico é a idade. A média de idade das mulheres participantes de estudos clínicos randomizados foi semelhante a encontrada neste estudo, variando de 46 a 49 anos. Entretanto, os autores apresentam a média de idade para todas as participantes, sem distinção entre as que apresentaram ou não neutropenia durante $o$ tratamento ${ }^{(8-9,12,14)}$.

Os resultados encontrados no presente estudo apontaram a ocorrência de diminuição de GB na progressão do tratamento quimioterápico, fato condizente com o estudo realizado no Reino Unido, com mulheres que receberam o tratamento quimioterápico adjuvante por câncer de mama, onde encontraram que $29 \%$ delas apresentaram, pelo menos, um episódio de neutropenia durante o tratamento. Os autores classificaram os eventos de neutropenia, de acordo com o protocolo de

\section{REFERÊNCIAS}

1. Dawood S, Broglio K, Gonzalez-Angulo AM, Buzdar AU, Hortobagyi GN, Giordano SH. Trends in survival over the past two decades among white and black patients with newly diagnosed stage IV breast cancer. J Clin Oncol. 2008;26(30):4891-8.

2. Moore S. Managing treatment side effects in advanced breast cancer. Semin Oncol Nurs. 2007;23(4 Suppl 2):S23-S30.

3. Gozzo TO. Toxicidade ao tratamento quimioterápico em mulheres com câncer de mama [tese]. Ribeirão Preto: Universidade de São Paulo, Escola de Enfermagem;2008.

4. Lyman GH, Lyman $\mathrm{CH}$, Agboola O. Risk models for predicting chemotherapy-induced neutropenia. Oncologist. 2005;10(6):427-37.

5. Rivera E, Haim Erder M, Fridman M, Frye D, Hortobagyi GN. First-cycle absolute neutrophil count can be used to improve chemotherapy-dose delivery and reduce the risk of febrile neutropenia in patients receiving adjuvant therapy: a validation study. Breast Cancer Res. 2003;5(5):R114-20.

6. Pettengell R, Schwenkglenks M, Leonard R, Bosly A, Paridaens $\mathrm{R}$, Constenla M, et al. Neutropenia occurrence and predictors of reduced chemotherapy delivery: results from the INC-EU Prospective observational European neutropenia study. Support Care Cancer. 2008;16(11):1299-309.

7. National Cancer Institute. Common Toxicity Criteria for Adverse Events [Internet]. Bethesda (MD); 2010 [Version 4.0; cited 2010 May 27]. Available from: http:// safetyprofiler-ctep.nci.nih.gov/CTC/CTC.aspx

8. Amat S, Bougnoux P, Penault-Llorca F, Fétissof F, Curé $\mathrm{H}$, Kwiatkowski F, et al. Neoadjuvant docetaxel for operable breast cancer induces a high pathological response and breast-conservation rate. Br J Cancer. 2003;88(9):1339-45.

9. Espinosa E, Morales S, Borrega P, Casas A, Madroñal C, Machengs I, et al. Docetaxel and high-dose epirubicin as quimioterapia aplicado. Nos protocolos que utilizaram antraciclinas, as mulheres mostraram $28 \%$ de neutropenia e $8,5 \%$ necessitaram de hospitalização. Concluíram que com a progressão do tratamento quimioterápico, os eventos neutropênicos parecem aumentar ${ }^{(15)}$.

\section{CONCLUSÕES}

Os resultados deste estudo mostram que, nos protocolos utilizados, a neutropenia tanto na neoadjuvância como na adjuvância, ocorreu a partir do segundo ciclo e manteve-se durante o tratamento.

A comparação entre as mulheres que tiveram neutropenia com aquelas que não, foi estatisticamente significante e mostrou que, na neoadjuvância, os valores de GB no ciclo dois foram os de maior gravidade. Além disso, na neoadjuvância foram registrados dois óbitos por neutropenia febril.

Assim, os resultados deste estudo apontam a necessidade de que estudos futuros acompanhem estas mulheres, em longo prazo, para que as toxicidades tardias do tratamento quimioterápico sejam avaliadas.

Apontam também para a importância da elaboração e implementação de protocolos e cuidados de enfermagem para mulheres com câncer de mama com a finalidade de avaliação dos eventos adversos e manejo mais adequado dos mesmos.

neoadjuvant chemotherapy in locally advanced breast cancer. Cancer Chemother Pharmacol. 2004;54(6):546-52.

10. Hirano A, Shimizu T, Imamura $H$, Watanabe $O$, Kinoshita J, Okabe T, et al. The combination of epirubicin plus docetaxel as neoadjuvant chemotherapy in locally advanced breast cancer. Anticancer Res. 2006;26(1B): 581-4.

11. Lück HJ, Thomssen C, Untch M, et al. Multicentric phase III study in first line treatment of advanced metastatic breast cancer (ABC). Epirubicin/paclitaxel (ET) vs epirubicin/cyclophosphamide (EC). A study of the AGO Breast Cancer Group. Proc Am Soc Clin Oncol. 2000;19 (73a): Abstract 280.

12. Ramaswamy B, Povoski SP, Rhoades C, Allen J, Hauger $M$, Young D, et al. Phase II trial of neoadjuvant chemotherapy with docetaxel followed by epirubicin in stage II/III breast cancer. Breast Cancer Res Treat. 2005;93(1):67-74

13. Jones RL, Walsh G, Ashley S, Chua S, Agarwal R, O'Brien $\mathrm{M}$, et al. A randomised pilot Phase II study of doxorubicin and cyclophosphamide (AC) or epirubicin and cyclophosphamide (EC) given 2 weekly with pegfilgrastim (accelerated) vs 3 weekly (standard) for women with early breast cancer. Br J Cancer. 2009;100(2):305-10.

14. Piccart MJ, Di Leo AD, Beauduin M, Vindevoghel A, Michel J, Focan C, et al. Phase III trial comparing two dose levels os epirubicin combined with cyclophosphamide, cyclophosphamide, methotrexate, and fluoracil in nodepositive breast cancer. I Clin Oncol. 2001;19(12):3103-10.

15. Leonard RC, Miles D, Thomas R, Nussey F; UK Breast Cancer Neutropenia Audit Group. Impact of neutropenia on delivering planned adjuvant chemotherapy: UK audit of primary breast cancer patients. Br J Cancer. 2003;89(11):2062-8. 